\title{
Propuesta de Estudio de la Red de TELEMEDICINA para el Departamento del Huila
}

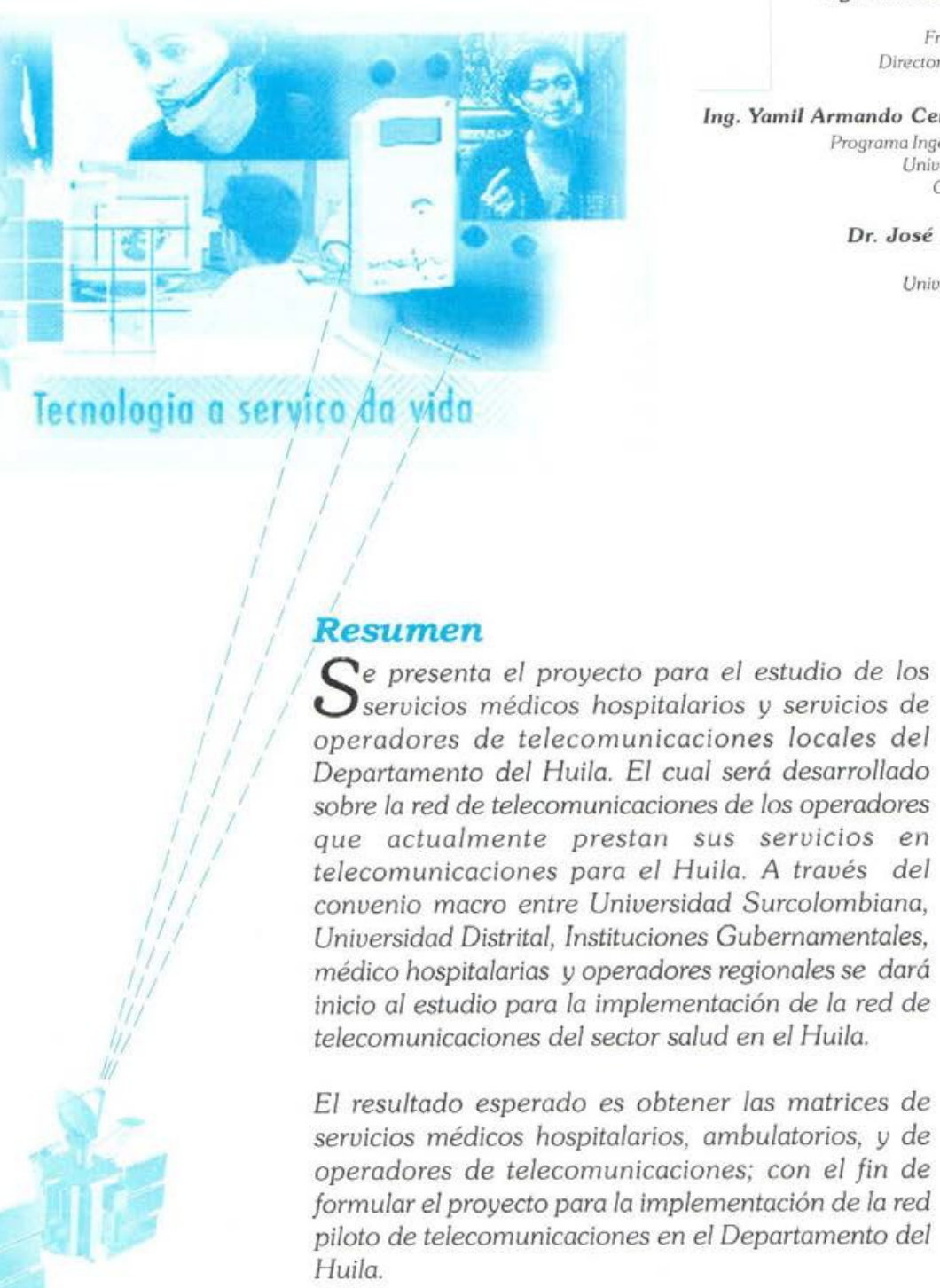

h Aparicio Pico Universidad Distrital Francisco José de Caldas rectora General del proyecto

ando Cerquera Rojas. Esp alngenieniería Electrónica Universidad Surcolombiana Codirector del proyecto Domingo Alarcón Facultad de Salud Universidad Surcolombiana biana 


\section{Introducción}

La telemedicina es hoy en día una herramienta dentro del avance tecnológico actual, que busca ampliar la cobertura de los servicios médicos hospitalarios al utilizar de manera correcta los avances tecnológicos en los sistemas de telecomunicaciones, la informática y los servicios multimedia; logrando el bienestar general, especialmente de los sectores sociales menos favorecidos. Las instituciones hospitalarias del primero, segundo y tercer nivel, ampliarán de manera preponderante la cobertura, la calidad del servicio y la calidad de vida (ANG99).

(N) El proyecto Telemedicina Neiva 2002, presentado y avalado por la Universidad Surcolombiana,a través de sus entes académicos y administrativos, tiene dentro de su plan estratégico cubrir la investigación. el desarrollo cientifico- tecnológico y la extensión. Con ello se evidencia el gran compromiso social de la Universidad, a través de la línea de investigación en Telemedicina, coordinada y dirigida por tres docentes de planta que integran las Facultades de Ingenieria y Medicina de la USCO; dos docentes de la Facultad de Ingenieria de la Universidad Distrital; además de un numeroso grupo de estudiantes de pregrado y postgrado de estas facultades.

A través del presente proyecto encaminado a evaluar los servicios del sector salud y de telecomunicaciones para el Departamento del Huila, se busca articular las prestaciones de los sectores anteriormente mencionados con el propósito de llevar a feliz tèrmino la prueba piloto de la Red de Telemedicina del Huila.

\section{Aspectos Básicos del Proyecto Telemedicina - Huila}

\section{Planteamiento del Problema}

Debido a la Problemática que afronta la Sociedad Colombiana en el sector de la salud, y ante la falta de servicios médico- asistenciales y médico-hospitalarios, se requiere ampliar la cobertura a los sectores menos favorecidos para cubrir la gran demanda de servicios, que permitan el acceso a todos los estratos socioeconómicos del Departamento. Es asi. como la Telemedicina se presenta como una buena alternativa para integrar los servicios médicos y poder resolver problemas neurálgicos como son:

a. El desarrollo social: teniendo en cuenta que la salud es uno de los mayores factores de incidencia en el desarrollo humano.

b. Los costos en los servicios médicos, de consulta o de diagnóstico son elevados, y el tiempo requerido en la integración de especialistas, alrededor de la solución de problemas específicos es lento.

c. En el momento no existe un diagnóstico real sobre los servicios requeridos en el área de telemedicina, razón suficiente para iniciar un trabajo de campo que establezca la situación actual de servicios médicos y la demanda real; asi como la posibilidad de conocer a corto, mediano y largo plazo, cuáles serian los costos de inversión que permitirian dar soluciones a los problemas de cobertura.

d. La socialización del conocimiento alrededor de la medicina, es uno de los valores que lleva al éxito de soluciones efectivas en el sector salud; por tal motivo es necesario desarrollar un plan de alfabetización en dicho campo, en los sectores gubernamental y educativo.

\section{Objetivo General}

Diseñar, implementar e integrar la red de servicios de telemedicina y de los sistemas de información en los diferentes niveles de atención en salud en el Departamento del Huila, evaluando las plataformas existentes ofrecidas por los operadores de Telecomunicaciones locales, para los servicios de voz, datos y video. 


\section{Objetivos Específicos}

* Establecer la matriz de integración de los servicios entre IPS públicas de los diferentes niveles de atención en salud, facultativos y administrativos para el Departamento del Huila, tanto en el sector privado como público en las entidades que participen del proyecto.

ss Evaluar la plataforma existente de servicios de operadores locales de Telecomunicaciones que participen, asi como la transferencia tecnológica necesaria para el desarrollo de la Red de Telemedicina del Departamento del Huila.

or Llevar a las diferentes zonas o regiones del Departamento especialidades y/o servicios médicos que por su naturaleza no están disponibles en todos estos lugares.

* Formular el proyecto para la Red Piloto de Telemedicina del Departamento del Huila.

* Diseñar la plataforma para adelantar la prueba piloto y realizar el convenio macro entre entidades que participan en la red piloto de Telemedicina del Departamento del Huila.

* Diseñar un plan de sensibilización sobre el impacto y desarrollo de la Telemedicina, en el sector salud y gubernamental.

of Posicionar al Departamento a la vanguardia tecnológica en la prestación de servicios de salud, contribuyendo con una mayor cobertura y una mejor calidad del servicio.

* Proporcionar escenarios de capacitación interactiva que contribuyan al mejoramiento del conocimiento y de las habilidades diagnósticas del cuerpo médico que pertenece a todos los centros asistenciales de la región.

os Implementar los sistemas de información en salud en red para el Departamento del
Huila con énfasis en los sistemas de vigilancia en salud pública.

Areas del Conocimiento en el Desarrollo del Proyecto

Telecomunicactones: La telemedicina esta ligada directamente con la transmisión de datos médicos (texto, imágenes, voz) entre los diferentes centros asistenciales. La clase de enlace de comunicaciones está en función del tipo de dato a transmitir, los cuales dependen fundamentalmente de la especialidad médica, con base en este requerimiento se debe definir el ancho de banda que satisfaga la necesidad especifica, permitiendo una buena velocidad de transmisión, calidad del dato y oportunidad en la prestación del servicio.

Informática: Nos ofrece un grupo de herramientas basadas en recursos computacionales, sistemas de información y redes de computadoras, que facilitan la prestación del servicio en salud. La computación y el procesamiento de datos están relacionados con la telemedicina como soporte para el manejo de historias clinicas. registro de ayudas diagnósticas, estadísticas, control de inventarios, estado y mantenimiento de los equipos de laboratorio y de ayudas diagnósticas.

Servicios Médicos: Los tipos de servicios de asistencia médica se definen con base a la estructura de prestación de servicios de salud. Para cada uno de los servicios se debe tener en cuenta el control de calidad, los aspectos economicos, y las condiciones legales, porque estos aspectos son claves para las sesiones de telemedicina.

\section{Contexto de la Telemedicina}

La telemedicina puede ser definida como la provisión del cuidado de la salud a través de una combinación de las telecomunicaciones y las tecnologias multimedia con médicos expertos. 
Teniendo en cuenta la propuesta, es necesario ubicar el contexto del proyecto en dos campos:

a. Caracteristicas especiales que se presentan en los servicios de Telemedicina. b. Plataforma de telecomunicaciones necesaria.

De otra parte, las comunicaciones necesarias en un sistema de Telemedicina son extensamente dependientes del tipo de servicio a utilizar. Estos servicios incluyen Teleconsulta, Telediagnóstico. Teleducación Médica, Teleradiologia, Teledermatología, Telecardiologia, Teleneuro-fisiologia, Telepsiquiatria, Acceso en Líneas a Base de Datos y Telemetria.

N

\section{z Requerimiento de comunicaciones}

Los sistemas de Telemedicina pueden requerir:

@- Dos o tres flujos de video simultáneos. Dos flujos de baja capacidad para videoconferencia con uno opcional de alta velocidad para videodiagnóstico. Para videoconferencia lo más conveniente son las normas H.261 (64Kb) $\mathrm{s}$ a $1.92 \mathrm{Mb} / \mathrm{s}, \mathrm{H} 263(15 \mathrm{~Kb} / \mathrm{s}$ a $34 \mathrm{Mb} / \mathrm{s})$ o MPEG$28(3-15 \mathrm{Mb} / \mathrm{s})$. en todos los casos de latencia el jitter ( la varianza en la rata de arribo en la red) de los flujos de video en tiempo real son criticos y deben ser minimizados (M0093-2).

$\square$ Dos o tres flujos simultáneos de audio. Dos de baja velocidad para teleconferencia y uno de alta velocidad para audio-diagnóstico, tal como el estetoscopio electrónico o sistema doppler de ultra sonido. Por ejemplo, un estetoscopio puede manejar una buena salida de audio digital a $128 \mathrm{~Kb} / \mathrm{s}$ usando un algoritmo Dolby AC-2.Basic G.711 a $56 \mathrm{~Kb} / \mathrm{s}$ es ruidoso y considerado inaceptable para consulta médica. G722 (48-64Kb/s). G723 (5$6 \mathrm{~Kb} / \mathrm{s})$. G. $726(32 \mathrm{Hb} / \mathrm{s})$. G.728 (15Kb/s) son más aceptables para teleconferencia. Los flujos de audio-diagnóstico podrian requerir audio con calidad de C.D., tal como audio MPEG-1 capa 2 (32-256Kb/s o Dolby AC-3 (93-768 Kb/s).

* Las imágenes son generalmente transmitidas en una sola dirección y son de alto volumen (10-526Mbyte/transferencia), la transferencia puede ser dinámicamente habilitada y deshabilitada, de acuerdo a su ancho de banda.

Una imagen de Rayos X, puede ser digitalizada con resolución de $2048 * 2048$ pixeles y $126 \mathrm{~b} /$ pixel con capacidad pixel almacenado en 2 bytes. $\mathrm{Si}$ el máximo retardo aceptado es de 10 s/imagen, un ancho de banda de al menos $7 \mathrm{Mb} / \mathrm{s}$ se requerirá para transferir la imagen dentro de ese limite, ignorando el sobreflujo y tráfico de la red. A pesar del flujo de video y audio, la latencia es menos critica para transferir imagen, y el jitter es irrelevante.

4. Registro médicos. La transferencia electrónica de los registros médicos es principalmente transmision unilateral de texto. La transmision de texto requiere bajo ancho de banda, la latencia no es critica, y el jitter es irrelevante.

\section{Beneficios del Proyecto}

$\Leftrightarrow$ Evita trasladar de un lugar a otro a un paciente para recibir una consulta médica, o simplemente para analizar un caso de dificil resolución.

e Permite en forma inmediata, poner en práctica las normas médicas de atención a escala regional.

* La consulta médica se extiende más allá, porque especialista y paciente pueden interactuar sin necesidad de estar fisicamente en el mismo lugar. Ampliando el acceso a especialidades médicas remotas como soporte de la asistencia sanitaria.

(*) Establecer interconsultas con personal médico altamente especializado a través de un sistema de referencia electrónica de pacientes, evitando traslados innecesarios de los pacientes a los centros de mayor complejidad.

* Aporta a la educación médica, a la administración de salud pública y a cada uno de las especialidades médicas.

c Permite la transmisión de cualquier tipo de información que requiera un proceso de digitalización. 
* Facilita la visualización y diálogo oportuno e inmediato entre personas o grupos de personas, permitiendo la interacción entre los participantes.

* Permite en forma remota compartir facilidades a bases de datos, o tecnologias multimediales.

« Se tiene la posibilidad de interconectar el sistema regional con redes similares. permitiendo acceso regional, nacional e internacional, ofreciendo las mismas facilidades para todos los usuarios, eliminando las barreras geográficas y ofreciendo al personal de áreas remotas el acceso a la información actualizada.

* Reducción de costos por los conceptos de transporte, gastos de viaje, combustible, tiempo, del personal médico, paramédicos o de los pacientes y sus familiares.

or A través de las sesiones de monitoreo y evaluación es posible medir el desarrollo de los objetivos institucionales y la ejecución de los diferentes programas que se llevan a cabo en cada zona.

$\Leftrightarrow$ Oportunidad en la resolución y terapéutica para el paciente: y disminución de remisiones.

Las aplicaciones de la telemedicina involucran diferentes tipos de datos: texto, imagen, audio, en esquemas de utilización en forma directa o en diferido. Entre las aplicaciones más importantes, tenemos las siguientes:

1. Teleradiologia: Transmisión electrónica de imágenes radiológicas de un sitio a otro con el propósito interpretación o consulta. Este servicio cubre los rayos $\mathrm{X}$, tomografia computarizada, imágenes de resonancia magnética, ultrasonido, y la termografia.

2. Telepatología: Es la práctica de la patología a distancia. Este servicio involucra la generación de opiniones diagnósticas sobre muestras en sitios remotos donde se usan computadores y tecnologias de comunicaciones.

3. Teledermatologia: Permite el traslado de imágenes para que los dermatólogos puedan observar el área afectada de la piel para hacer su diagnóstico.
4. Teleconsulta médica: Por medio del sistema de comunicaciones y/o a través de un sistema de video conferencia se puede practicar la teleconsulta, permitiendo el intercambio de información que sirva como ayuda diagnóstica y procedimental.

5. Tele-educación médica: Proporciona nuevas formas de aprendizaje, que enriquecen los conocimientos de los profesionales de la salud, sin restricciones de espacio y tiempo. Este servicio responde a las necesidades en materia de orientación y capacitación a distancia de profesionales de la salud que trabajan en puestos y centros de salud apartados o en zonas rurales.

6. Telecardiología: Esta aplicación permite a través de conexiones de la red de telecomunicaciones transferir electrocardiogramas a cardiólogos o centros de emergencia.

7. Teleneurofisiología: Es la transferencia en tiempo real de electroencefalogramas por medio de redes de telecomunicaciones, este servicio es exigente en ancho de banda y velocidades de transmisión.

8. Telepsiquiatría: Permite las consultas entre médicos de las instituciones de salud y psiquiatras por medio de videoconferencia en tiempo real.

9. Telefarmacia: Por medio de este servicio implementamos dispositivos automáticos de distribución de droga, previa prescripción médica.

10. Acceso en línea a Bases de Datos: Se permite intercambiar datos entre las instituciones de salud, podemos tener archivos sobre el resultado de un tratamiento. imágenes, correo para propósitos administrativos, boletines cientificos, archivos de pacientes, cartas de referencia, resultados de exámenes, acceso a bases de datos médicas especializadas, etc. 
11. Telemetrio: Para estudiar y supervisar las funciones fisiológicas humanas en un sitio remoto, de esta forma logramos telemonitorear los parámetros físicos y ambientales de los pacientes.

\section{Pretensiones del Proyecto}

\& Diseñar e implementar la red de telecomunicaciones del Hospital Universitario del Huila Hernando Moncaleano Perdomo.

* Diseñar e implementar la red Departamental de telecomunicaciones del sector de la salud.

* Realizar el análisis y diseño de un sistema de información que permita la consulta de historias clínicas en linea.

is Diagnosticar el equipo electrónico existente en el Hospital Universitario Hernando Moncaleano Perdomo. planteando un plan general para el mantenimiento preventivo de los mismos.

or Realizar el análisis y diseño del sistema de inventario de equipos electromédicos, que incluye datos como: características, fabricante, localización. uso, fecha de compra, último mantenimiento, costo, interfase de comunicaciones, categoria, serial, persona responsable, proveedor, garantia, estado, etc.

a Realizar el análisis y diseño del sistema de información para la vigilancia del sector salud.
* Desarrollo del sistema de vigilancia en salud pública para el Departamento del Huila.

\section{Conclusiones y Recomendaciones}

\section{Son varias las novedades que se proyectan con el} desarrollo del proyecto:

1. La matriz de integración de servicios médicohospitalarios, por cuanto en ella se refleja la potencialidad real de servicios de telemedicina para el Departamento del Huila.

2. Posibilidades de utilizar servicios de valor agregado en implantación de servicios de telemedicina ofrecido por los operadores de telecomunicaciones locales.

3. Diseño de la plataforma para desarrollo de la prueba piloto que dará la pauta de la implementación total de la red.

4. Realización de convenios interinstitucionales, para apoyo económico, logístico, técnico y cientifico del proyecto.

5. Se abre la posibilidad de inversión extranjera y nacional para el desarrollo y montaje de la red de Telemedicina del Departamento del Huila.

6. Se crea la linea de investigación en Telemedicina en la Universidad Surcolombiana con la participación de un grupo multidisciplinario de profesionales.

7. Se plantea la posibilidad de desarrollar múltiples proyectos que serán manejados por egresados de programas que ofrecen tanto a la Universidad Surcolombiana y la Universidad Distrtial. 


\section{Bibliografía}

1. (ANG99) Aparicio Edith, Angulo Ch. Jaime Gonzalez R. Ivan F. Redes de Telemedicina, Universidad Distrital. Francisco José de Caldas.

2. (M0093) M. Moore. Elements of Succes in Telemedicine Projects, Graduate School of Library and Information Science, University of Texas at Agustin,1993.

3. (PROO0) L:E: Aparicio, J:H: Angulo, G.R. Ivan, J.A. Benitez «proyecto Telemedicina Bogotá 2000" Universidad Distrital Francisco José de Caldas, 2000.

4. Pradhan M., «Important Concepts in Telemedicine», in Health Informatics, Melbourne Australia, 1994.
5. Lin J., "Current Developments in Telemedicine», IEEE engineering in Medicine and Biology, vol. 18, n. 4,pp.27, 1999.

6. Grigsby $J_{\text {., Lindsey B., }}$ "Telecommunications Technology, Health Services, and Technology Assesmentw, Internal Report, University Colorado Health Sciences Center, Denver, 1998.

7. Holle R., Zahlmann G., «Evaluation of Telemedical Services", IEEE Transactions on Information Technology in Biomedicine, vol.3,n.2,pp.8491,1996. 